\title{
Transcranial Doppler Ultrasonography
}

National Cancer Institute

\section{Source}

National Cancer Institute. Transcranial Doppler Ultrasonography. NCI Thesaurus. Code C122930.

A diagnostic technique that uses pulsed Doppler ultrasound to measure the velocity of blood flow through the major blood vessels of the brain. 